\title{
First Demonstration of a Broadband 37-cell Hollow Core Photonic Bandgap Fiber and Its Application to High Capacity Mode Division Multiplexing
}

\author{
Y. Jung, ${ }^{1 *}$ V.A.J.M. Sleiffer, ${ }^{2}$ N. Baddela, ${ }^{1}$ M.N. Petrovich, ${ }^{1}$ J.R. Hayes, ${ }^{1}$ N.V. Wheeler, ${ }^{1}$ D.R. Gray, ${ }^{1}$ \\ E. Numkam Fokoua, ${ }^{1}$ J.P. Wooler, ${ }^{1}$ N.H.-L. Wong, ${ }^{1}$ F. Parmigiani, ${ }^{1}$ S.U. Alam, ${ }^{1}$ J. Surof, ${ }^{3}$ M. Kuschnerov, ${ }^{4}$ \\ V.Veljanovski, ${ }^{4}$ H.de Waardt, ${ }^{2}$ F. Poletti, ${ }^{1}$ and D.J. Richardson ${ }^{1}$ \\ ${ }^{1}$ Optoelectronics Research Centre, University of Southampton, Southampton, SO17 1BJ, UK \\ ${ }^{2}$ COBRA institute, Eindhoven University of Technology, The Netherlands \\ ${ }^{3}$ Technische Universität München, Munich, Germany \\ ${ }^{4}$ NSN Optical GmbH, Munich, Germany \\ *ymj@orc.soton.ac.uk
}

\begin{abstract}
We report fabrication of the first low-loss, broadband 37-cell photonic bandgap fiber. Exploiting absence of surface modes and low cross-talk in the fiber we demonstrate mode division multiplexing over three modes with record transmission capacity.

OCIS codes: (060.4005) Microstructured fiber; (060.0060) Fiber optics and optical communications.
\end{abstract}

\section{Introduction}

Hollow core photonic bandgap fibers (HC-PBGFs) have long been viewed as interesting candidates for data transmission because of their low nonlinearity and potential for ultralow loss. However, issues associated with surface modes (SMs), which substantially reduce the usable fiber bandwidth, along with concerns about the number of higher order modes have discouraged work in this area, despite losses as low as $1.7 \mathrm{~dB} / \mathrm{km}$ reported in 2004 [1]. Recently, however, 19-cell (19c) HC-PBGFs with wide bandwidths $(>150 \mathrm{~nm})$ and a few dB/km loss [2, 3] have been demonstrated to enable high capacity, low-latency single-mode data transmission at both $1.5 \mu \mathrm{m}$ and $2 \mu \mathrm{m}[4,5]$.

A possible solution to further reduce surface scattering -the primary loss mechanism in these fibers- is to decrease the optical field overlap with the glass, e.g. by enlarging the core size from 19 to 37 cells (37c). HC-PBGFs with bigger cores, however, present a number of challenges, including stricter fabrication tolerances, difficulties associated with a potentially higher number of surface modes and an increased number of core guided modes [6], which are more narrowly spaced and thus more susceptible to perturbation-induced coupling. In principle, mode coupling along the fiber can be unraveled using Multiple Input Multiple Output (MIMO) techniques, so that separate modes can be used to transmit independent channels, making the intrinsic multi-mode nature of HC-PBGFs a feature to exploit rather than a problem. However, the very first experiments of mode division multiplexing (MDM) in HCPBGFs have raised the question as to whether high capacity MDM might be possible at all [7, 8].

In this work, we report the fabrication of the first ever low-loss, wide-bandwidth 37c HC-PBGF, a fundamental step towards achieving ultralow-loss HC-PBGFs. The fiber, obtained by applying similar core-surround engineering as previously demonstrated for $19 \mathrm{c}$ fibers [2,3], has a low minimum fundamental mode loss of $3.3 \mathrm{~dB} / \mathrm{km}$ at 1550 $\mathrm{nm}$ and a wide $3-\mathrm{dB}$ bandwidth of $\sim 85 \mathrm{~nm}$ centered in the C-band. By using mode multiplexing techniques, we characterize in detail the fiber's modal properties, showing that it supports significantly less modes than would have been expected and that at least the $\mathrm{LP}_{01}$ and $\mathrm{LP}_{11}$ modes have properties compatible with MIMO-based MDM.

To prove it, we report the first transmission of polarization and wavelength division multiplexed (DWDM) signals over 3 modes in such a fiber. Using 96 DWDM channels, 3 MDM modes and a 16-level quadrature amplitude modulation (16-QAM) we achieve a total data rate of $73.7 \mathrm{~Tb} / \mathrm{s}$, which sets a new transmission record for HC-PBGFs.

\section{Fabrication and characterization of 37-cell HC-PBGF}

The HC-PBGF (shown in the inset of Fig.1a) was fabricated by omitting 37 capillaries in the stack and by accurately controlling differential pressures during both drawing stages. Its cladding pitch and relative hole size $(4.4 \mu \mathrm{m}$ and 0.97 , respectively) provide a $\sim 300 \mathrm{~nm}$ wide bandgap. Although controlling SM position and number is more challenging than with 19c fibers, we managed to achieve a fiber with only two groups of SMs, which we successfully located at either sides of the $\mathrm{C}$-band, providing an overall $\sim 85 \mathrm{~nm}$ wide low loss bandwidth (Fig. 1 e).

Despite a large core diameter of $37 \mu \mathrm{m}$, which is required for low loss operation but is in principle able to support as many as 80 core modes, $\mathrm{S}^{2}$ measurements indicate that only 16 modes divided in 5 mode groups (even fewer than in a previously reported 19c HC-PBGF [2]) are guided with low loss in our fiber. $\mathrm{S}^{2}$ cutback analysis (traces at $9 \mathrm{~m}$ and $2 \mathrm{~m}$ shown in Fig. $1 \mathrm{~b}$ ) reveals that modes beyond the $\mathrm{LP}_{02}$ experience high propagation losses (estimated at $\geq 6$ $\mathrm{dB} / \mathrm{m}$ ) likely due to coupling to lossier cladding modes; a more detailed investigation is still underway. 
(a)

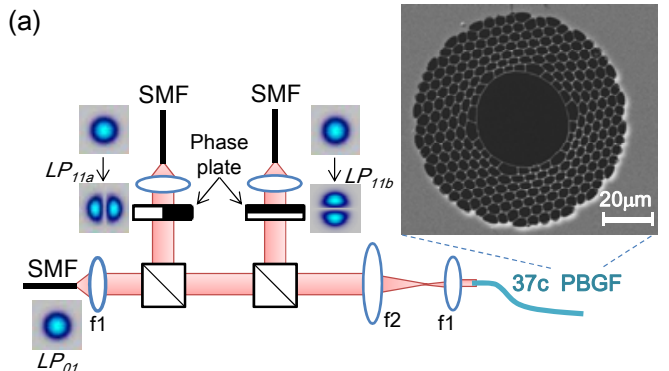

(c)

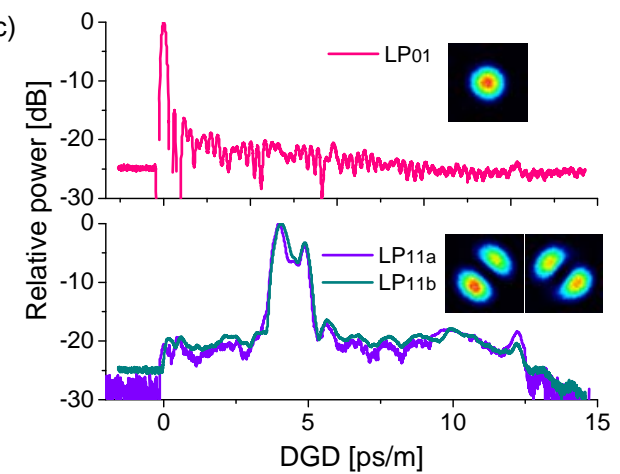

(e)

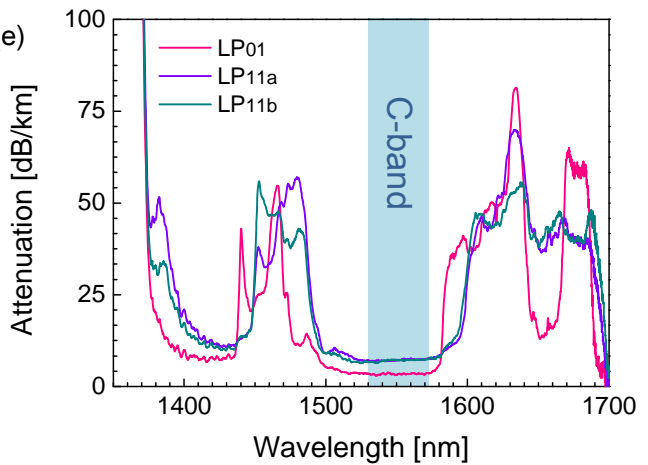

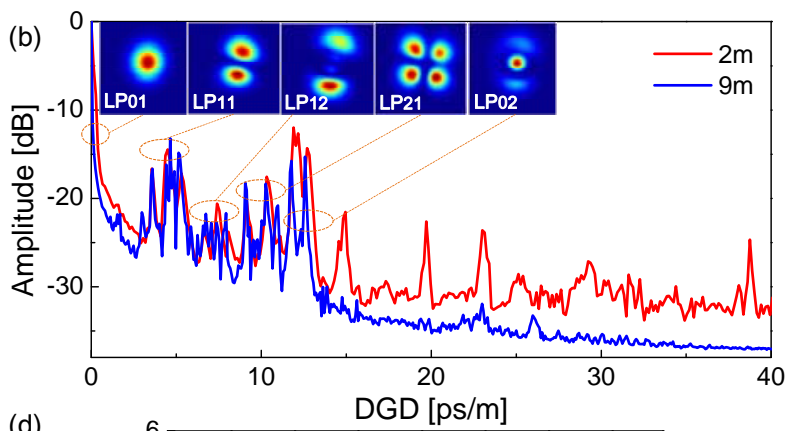

(d)

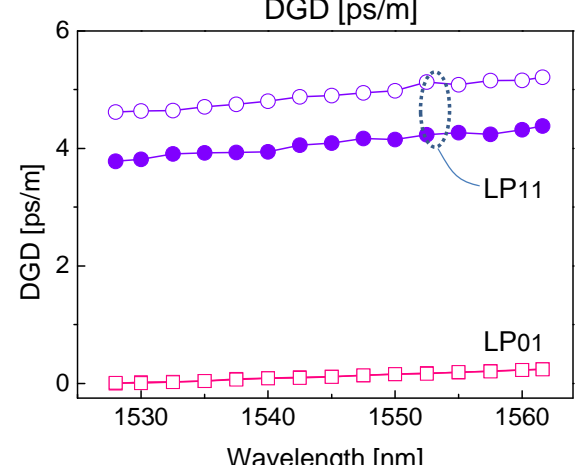

(f)

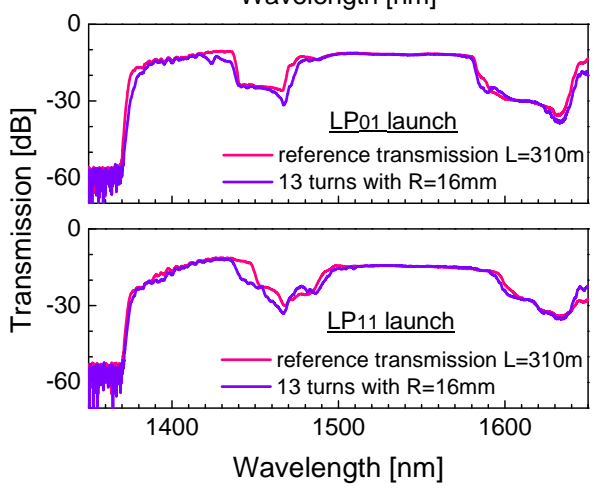

Fig. 1(a) Schematic of three-mode $\left(\mathrm{LP}_{01}, \mathrm{LP}_{11 \mathrm{a}}, \mathrm{LP}_{11 \mathrm{~b}}\right)$ mode multiplexer and 37c HC-PBGF SEM image (b) $\mathrm{S}^{2}$ analysis of modal content ( $9 \mathrm{~m}$ and $2 \mathrm{~m}$ fiber lengths) (c) ToF measurement for pure $\mathrm{LP}_{01}, \mathrm{LP}_{11 \mathrm{a}, \mathrm{b}}$ modes and (d) DGD as a function of wavelength (e) Transmission loss of $\mathrm{LP}_{01}$ and $\mathrm{LP}_{11 \mathrm{a}, \mathrm{b}}$ modes from a $310 \mathrm{~m}$ to $20 \mathrm{~m}$ cutback and (f) Bend loss performance of the 37c HC-PBGF.

In this work we focus on the characterization of three lowest order spatial modes $\left(\mathrm{LP}_{01}, \mathrm{LP}_{11 \mathrm{a}}, \mathrm{LP}_{11 \mathrm{~b}}\right)$, which are predicted to have the lowest propagation losses [9], and present a detailed description of their properties. To selectively launch the three individual modes we employed the phase-plate based mode multiplexer (MMUX) illustrated in Fig.1a. Light from a supercontinuum (SC) source was split in three paths, encoded in the required mode patterns and coupled into $310 \mathrm{~m}$ of 37c HC-PBGF via a telecentric lens arrangement. The SC laser provided a high average power which was required to compensate for the high MMUX insertion losses.

Time-of-flight (ToF) measurements [2] using sub-ps pulses from a mode-locked laser at $1550 \mathrm{~nm}$ and a $10 \mathrm{GHz}$ bandwidth sampling oscilloscope were employed during the modal characterization to optimize the launch of each individual mode. Typical results are shown in Fig.1c: the DGD of the $\mathrm{LP}_{11}$ and $\mathrm{LP}_{21}$ modes was estimated at 4-5ps/m and $10-12 \mathrm{ps} / \mathrm{m}$, respectively, in good agreement with $\mathrm{S}^{2}$ results. Note that DGD values are roughly $50 \%$ smaller than in a $19 \mathrm{c}$ fiber $[2,3]$. The extinction ratio provided at launch by our MMUX was about $20 \mathrm{~dB}$. For $\mathrm{LP}_{11}$ mode launch we observed strong coupling between the two spatial orientations and a plateau of distributed scattering [10] of about $-20 \mathrm{~dB}$ to both $\mathrm{LP}_{01}$ and $\mathrm{LP}_{21}$ modes. Modal cross-coupling is expected to decrease as the fiber loss is reduced since the two effects have the same underlying physical mechanism in HC-PBGFs. The wavelength dependence of the DGD (Fig. 1d) indicates chromatic dispersion around 7 and $18 \mathrm{ps} / \mathrm{nm} / \mathrm{km}$ for $\mathrm{LP}_{01}$ and $\mathrm{LP}_{11}$ modes, respectively.

Through a careful cutback from $310 \mathrm{~m}$ to $20 \mathrm{~m}$ the mode-specific spectral loss (shown in Fig. 1e) was measuredto the best of our knowledge the first such measurement in a HC-PBGF. All curves present two distinct peaks due to SM anticrossings which, as predicted from theory but never before shown experimentally, are mode dependent and 
shifted $\sim 10 \mathrm{~nm}$ to longer wavelengths for the $\mathrm{LP}_{11}$ modes as compared to the $\mathrm{LP}_{01}$ mode. The minimum loss values for $\mathrm{LP}_{01}$ and $\mathrm{LP}_{11 \mathrm{a}, \mathrm{b}}$ are $3.3 \pm 0.8$ and $7.4 \pm 0.8 \mathrm{~dB} / \mathrm{km}$, respectively, at $1550 \mathrm{~nm}$. The higher loss of the $\mathrm{LP}_{11}$ modes derives from a higher overlap with the air-glass interfaces which causes stronger scattering, and the value of the differential loss between $\mathrm{LP}_{01}$ and $\mathrm{LP}_{11}$ is in good agreement with numerical predictions [9]. Despite its large core, the fiber is still very robust to bending. Fig. 1f shows the spectral change in transmission after a short section near the output end of the HC-PBGF was coiled into 13 loops of $16 \mathrm{~mm}$ radius. While some bend-induced loss increase (and associated bandwidth decrease) was observed at or near the SMs, no measurable change was observed for any core mode in their respective low loss region, providing further evidence of the importance of suppressing SMs.

\section{Demonstration of Mode Division Multiplexed transmission}

Exploiting the excellent modal qualities of this novel 37c HC-PBGF we demonstrated the feasibility of large capacity transmission employing the MDM transmission setup described in [11]. The WDM system included 96 external cavity lasers running on a $50 \mathrm{GHz}$ ITU grid in the extended C-Band (1528.77-1566.72 nm), modulated with $256 \mathrm{~Gb} / \mathrm{s}$ dual-polarization 16-level quadrature amplitude modulation (DP-16QAM). The input signal was split into three equal-power replicas, delayed with respect to one another for de-correlation and mode-division multiplexed using the MMUX of Fig.1a. The resulting $73.7 \mathrm{~Tb} / \mathrm{s}$ WDM-MDM signal was transmitted over $310 \mathrm{~m}$ of HC-PBGF, after which the modes were demultiplexed. The channel under test (CUT) was selected using $50 \mathrm{GHz}$ optical filters and received with three coherent receivers connected to time-synchronized digital sampling scopes; the recorded data was processed offline using a $6 \times 6$ MIMO digital signal processing algorithm to decouple the signals. Fig. 2 shows the bit error rate performance after transmission for all 96 channels, the received spectrum at the $\mathrm{LP}_{01}$ port and the received constellation for the channel running at $1550.12 \mathrm{~nm}$. As can be observed, all channels are well below the FEC-limit at $2.4 \times 10^{-2}$ [12], again providing compelling evidence of excellent fiber modal characteristics.
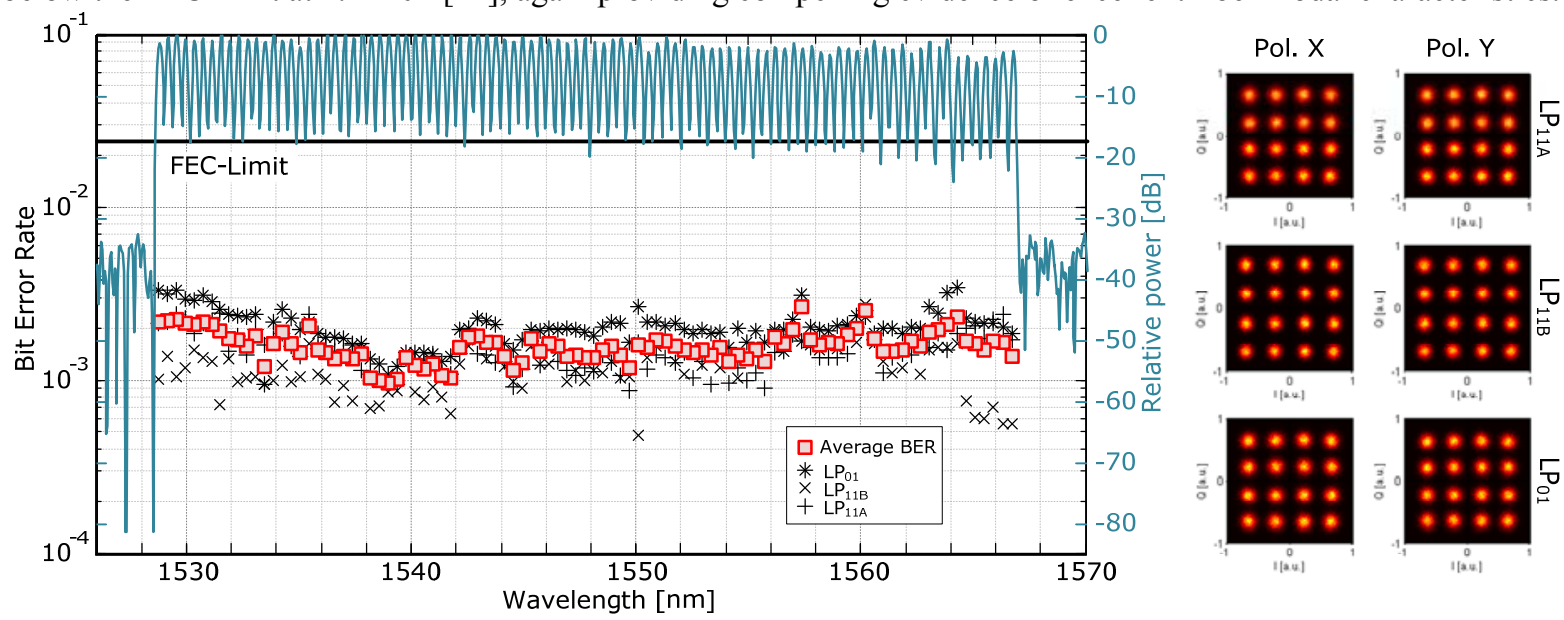

Fig. 2. Left: Bit-error rate of the demodulated signal after transmission over the $37 \mathrm{c}$ PBGF and the received spectrum at the $\mathrm{LP}_{01}$ port. Right: Received 16QAM constellations for the channel at $1550.12 \mathrm{~nm}$.

\section{Conclusions}

We have reported the first ever detailed modal characterization of a novel 37c HC-PBGF boasting a low minimum fundamental mode loss of $3.3 \mathrm{~dB} / \mathrm{km}$ at $1550 \mathrm{~nm}$ and an $85 \mathrm{~nm}$ wide $3-\mathrm{dB}$ bandwidth. The mode-dependent spectral and bend loss of its 3 lowest order modes were measured along with their propagation and cross coupling properties. Absence of SMs and low cross-talk are pre-requisites for high capacity MDM-DWDM transmission in a HC-PBGF, which we demonstrate here for the first time. Our total data rate of $73.7 \mathrm{~Tb} / \mathrm{s}$ sets a new transmission capacity record for HC-PBGFs. These results are an important step towards ultralow-loss transmission fibers and illustrate the intriguing possibility to employ MDM in such low nonlinearity, low latency fibers.

This work was supported by the European Communities $7^{\text {th }}$ Framework Programme under grant agreement 258033 (MODE-GAP).

\section{References}

[1] B. J. Mangan et al., Proceedings of OFC'04, pp. PDP24.

[3] F. Poletti et al, Nature Photonics 2013 (Accepted)

[5] M. N. Petrovich et al., Proceedings of ECOC'12, Th.3.A.5.

[7] J. Xu et al., Opt. Express, vol. 20, 12449-12456 (2012).

[9] E. Numkam et al., Opt. Express, vol. 20, 20980-20991 (2012).

[11] V.A.J.M. Sleiffer et al., Opt. Express, vol. 20, B428-B438 (2012).

[2] N. V. Wheeler et al., Proceedings of OFC'12, pp. PDP5A.2.

[4] V. Sleiffer et al., Proceedings of OFC'13, pp. OW1I.5.

[6] M. N. Petrovich et al., Opt. Express 16, 4337-4346 (2008)

[8] J. Carpenter et al., Proceedings of OFC'12, pp. JW2A.

[10] J.W.Nicholson, IEEE J. Sel. Top. Q. Electron 15, 61 (2009).

[12] D.A. Morero et al., IEEE Globecom (2011). 